Review Article

\title{
Is There a Place for Spinal Cord Stimulation in the Management of Patients with Multiple Sclerosis? A Systematic Review of the Literature
}

\author{
Alessandro Rapisarda $\left(\mathbb{D},{ }^{1}\right.$ Eleonora Ioannoni ${ }^{D},{ }^{2}$ Alessandro Izzo ${ }^{(D)},{ }^{1}$ \\ Manuela D'Ercole ${ }^{D},^{1}$ and Nicola Montano ${ }^{1}{ }^{1}$ \\ ${ }^{1}$ Department of Neuroscience, Neurosurgery Section, Fondazione Policlinico Universitario Agostino Gemelli IRCCS, \\ Università Cattolica del Sacro Cuore, Rome, Italy \\ ${ }^{2}$ Neurosurgical Intensive Care Unit, Fondazione Policlinico Universitario A. Gemelli IRCCS, Rome, Italy
}

Correspondence should be addressed to Nicola Montano; nicolamontanomd@yahoo.it

Received 9 March 2021; Accepted 9 April 2021; Published 20 April 2021

Academic Editor: Vadim Byvaltsev

Copyright (c) 2021 Alessandro Rapisarda et al. This is an open access article distributed under the Creative Commons Attribution License, which permits unrestricted use, distribution, and reproduction in any medium, provided the original work is properly cited.

\begin{abstract}
Objective. Spinal cord stimulation (SCS) is a minimally invasive technique mainly used to treat neuropathic pain associated with failed back surgery syndrome. However, this therapy has been utilized to treat other chronic painful conditions, such as pain associated with multiple sclerosis (MS). Nonetheless, the efficacy of SCS in MS patients has not been fully established. In fact, in most of SCS series, MS patients represent only a subset of a bigger cohort which comprises different causes of pain, motor disorder, and other functional limitations. The aim of our study was to systematically review the literature to evaluate the effectiveness of SCS in MS patients. Methods. A literature search was performed through different databases (PubMed, Scopus, and Embase) using the following terms: "multiple sclerosis," "spinal cord stimulation," and "dorsal column stimulation," according to PRISMA (Preferred Reporting Items for Systematic Reviews and Meta-analyses) guidelines. Results. A total of 452 articles were reviewed, and 7 studies were included in the present analysis. 373 MS patients were submitted to a stimulation trial, and 82 MS patients underwent a de novo implantation. 285/373 (76.4\%) of cases submitted to the SCS trial were enrolled for permanent stimulation. We found a long-lasting improvement in 193/346 (55.8\%) MS patients with motor disorders, in 90/134 (67.13\%) MS patients with urinary dysfunction, and in 28/34 (82.35\%) MS patients with neuropathic pain. The efficacy of SCS was higher for urinary dysfunction $(p=0.0144)$ and neuropathic pain $(p=0.0030)$ compared with motor disorders. Conclusions. Our systematic review evidences that SCS is effective in MS patients. Urinary dysfunction and pain symptoms seem to be most responsive to SCS. Further studies are needed to improve the patient selection and clarify the best timing to perform SCS in these patients.
\end{abstract}

\section{Introduction}

Motor disorders, neuropathic pain, and urinary dysfunction are the main causes of functional limitations in patients affected by multiple sclerosis (MS) [1-4]. All these symptoms may become unresponsive to traditional immune-modulating treatment or to medication for pain and may impact negatively on the quality of life of these patients [5]. Since the pioneering paper of Cook and Weinsten [6] who firstly submitted an MS patient to spinal cord stimulation (SCS) to treat an incoercible back pain observing a long-lasting pain relief, SCS has been used to treat the different MS-associated symptoms. Unfortunately, in most of the published papers, MS patients represent only a subset of a bigger cohort which comprises different causes of pain, motor disorder, and other functional limitations. Since few studies specifically focused on MS patients, the results of SCS in this subgroup are often ambiguous and contradictory. The aim of our study was to systematically review the literature on SCS in MS patients analysing the results of this technique on motor, pain, and urinary symptoms recovery. 


\section{Materials and Methods}

2.1. Inclusion Criteria and Measurement of Outcomes. This study was conducted in agreement with the PRISMA (Preferred Reporting Items for Systematic Reviews and Meta-analyses) guidelines statement [7]. Three medical databases (PubMed, Scopus, and Embase) were screened for eligible scientific reports. The key words "multiple sclerosis," "spinal cord stimulation," and "dorsal column stimulation" were used in any possible combination. The last search was launched in June 2020. Two reviewers (A.R. and E.I.) independently screened the abstracts and the references list. Any difference was solved by consensus with a third senior author (N.M.). Studies were included if they met the following criteria: (1) English prospective or retrospective studies on SCS in MS patients, (2) series with more than 5 patients, and (3) series which clinical data, outcome, and follow-up (FU) were clearly reported for each patient. As outcome variables, motor function, pain, and urinary dysfunction were evaluated. We considered a patient improved for a specific function if the authors reported an improvement for that function regardless of the evaluation scale used.

2.2. Statistical Analysis. Statistical analyses were done using StatView version 5 software (SAS Institute Inc.). Statistical comparison of categorical variables was performed by $\chi 2$ statistic, using the Fisher's exact test. Differences were considered significant at $p<0.05$.

\section{Results}

A total of 452 articles were identified and reviewed (Figure 1). Finally, 7 studies were included in the present investigation (Table 1) [8-14]. Overall, there were $373 \mathrm{MS}$ patients who were submitted to a SCS trial and 82 MS patients who underwent a de novo implantation. The mean age at implantation was $44.25 \pm 0.75$ years, and the mean FU was $44.40 \pm 25.00$ months. Out of the patients submitted to the SCS trial, 285/373 (76.4\%) were enrolled for permanent stimulation. Overall, a long-lasting improvement (at latest available FU) was observed in 193 out of $346(55.8 \%)$ MS patients with motor disorders, in 90 out of $134(67.13 \%)$ MS patients with urinary dysfunction, and in 28 out of $34(82.35 \%)$ MS patients with neuropathic pain. The efficacy of SCS was higher for urinary dysfunction $(p=0.0144)$ and neuropathic pain $(p=0.0030)$ compared with motor disorders (Table 2). These significant differences were confirmed in the subgroup of patients submitted to the SCS trial and then to the definitive implant (Table 2). In the subgroup of patients who underwent a de novo implantation (without the trial), a significant difference was maintained only for neuropathic pain (Table 2).

\section{Discussion}

MS is a chronic demyelinating disease determining a wide variety of neurological symptoms. MS has been reported as the most common cause of neurological disability in young adults [15], and its onset typically occurs between 20 and 40 years. The incidence in the female sex has been reported from two to three times higher than the male sex. The current incidence in Western Europe ranges between 2/ 100000 /years and 18.2/100000/years [16] and is constantly increasing. MS has been categorized into four distinct clinical subtypes: relapsing-remitting, secondary-progressive, primary progressive, and progressive relapsing [17]. However, all types of MS show a neuroaxonal dysfunction determining, among the others, weakness, visual impairment, bladder dysfunction, sensory impairment, fatigue, spasticity of the extremities, trigeminal neuralgia, and neuropathic pain, which is found in approximately $50 \%$ of MS patients [18-20]. These symptoms may become unresponsive to the medical management and may significantly worsen the quality of life of these patients $[2,5,21]$. SCS is a minimally invasive technique mainly used to treat the neuropathic pain associated with the failed back surgery syndrome and other neuropathic painful conditions. Effectiveness of SCS in MS patients has been thoroughly debated in literature without a definitive conclusion. No clear indication exists on which patients can benefit from SCS and on the best timing to perform this procedure in the history of these patients. This is probably because MS patients were included in SCS series only as a subgroup of a bigger cohort of patients. The aim of our systematic review was to analyse the results of SCS in MS patients on motor function, urinary dysfunction, and neuropathic pain taking into account only articles reporting MS patients in which the results were clearly presented. We found that SCS may improve all these symptoms in more than $50 \%$ of cases and that SCS showed a significantly higher efficacy for urinary symptoms and neuropathic pain compared with motor disorders. SCS has the great advantage of being a neuromodulation procedure that is nondestructive and reversible. The main shortcoming in MS patients was the noncompatibility of the previous SCS devices with magnetic resonance imaging (MRI) which prevented the regular follow-up of the disease. However, in the last few years, the improvement of technology overcame this problem because of the development of MRI-compatible systems [21]. Moreover new stimulation paradigms, such as the high frequency paradigm, could play a role in the management of MS patients [22]. Thus, in our opinion, SCS may represent a valid option for MS patients whose symptoms are not controlled by medications. Moreover, a better selection of cases (patients who mainly complain of neuropathic pain and urinary dysfunction) and the implementation of a stimulation trial before the definitive implantation may help in increasing the number of patients responding to this treatment, as we demonstrated in this systematic review. The most frequent SCS complications comprise system malfunctioning or breakage, wound or system infections, and epidural hematoma. However, from an initial rate of $65 \%$ of the implanted patients [9], these events became less frequent subsequently [14] probably due to the evolution of the implant technique. 


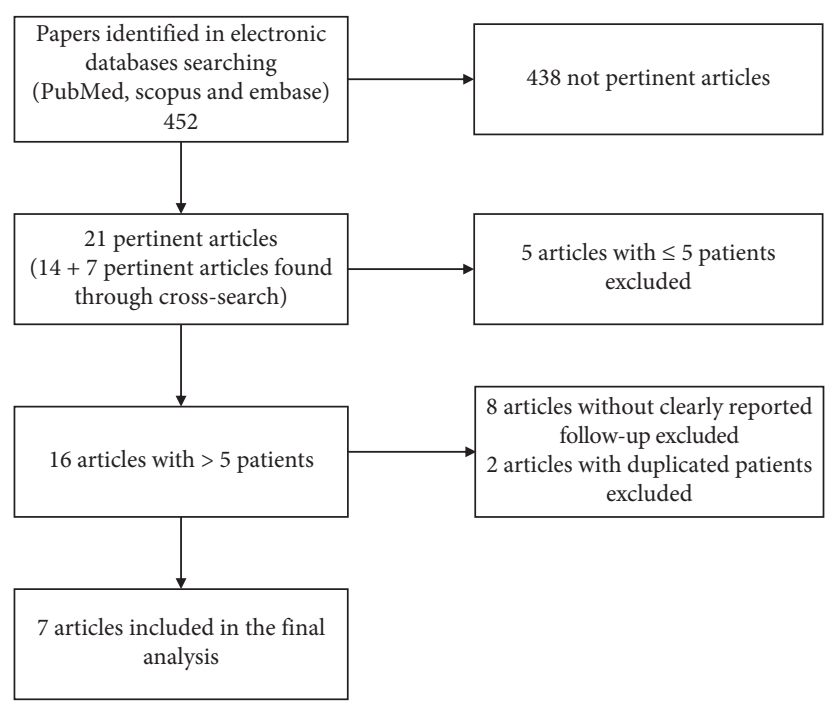

FIgURE 1: Flowchart of study selection.

TABLE 1: Studies included in the pooled statistical analysis.

\begin{tabular}{|c|c|c|c|c|}
\hline Author/year & $\begin{array}{c}\text { Patients submitted to } \\
\text { stimulation trial }\end{array}$ & $\begin{array}{c}\text { Patients with a definitive } \\
\text { implant }\end{array}$ & Type of the study & $\begin{array}{l}\text { Follow-up } \\
\text { (months) }\end{array}$ \\
\hline Rosen and Barsoum (1979) [8] & 9 & 9 & Retrospective & $6-37$ \\
\hline Young and Goodman (1979) [9] & 23 & 20 & Retrospective & 32 \\
\hline Illis et al. (1980) [10] & 19 & 10 & Retrospective & 24 \\
\hline Sigfried et al. (1981) [11] & 111 & 37 & Retrospective & $12-70$ \\
\hline Cook et al. (1981) [12] & 192 & 204 (192 posttrial + 12 de novo $)$ & Retrospective & 12 \\
\hline Waltz et al. (1987) [13] & None & 91 (de novo) & Retrospective & $6-120$ \\
\hline Kumar et al. (2006) [14] & 19 & 17 & Retrospective & 97.6 \\
\hline
\end{tabular}

TABLE 2: Number of MS patients improved after SCS according to the evaluated function.

\begin{tabular}{lcc}
\hline Function & Improvement (yes/no) & $p$ \\
\hline All patients & & \\
Motor disorders & $193 / 153$ & $p=0.0144$ \\
Urinary dysfunction & $90 / 44$ & $p=0.0030$ \\
Neuropathic pain & $28 / 6$ & \\
\hline Patients submitted to the trial and then implanted & \\
Motor disorders & $158 / 121$ & $p=0.038$ \\
Urinary dysfunction & $40 / 17$ & $p=0.044$ \\
Neuropathic pain & $15 / 4$ & \\
\hline Patients with de novo implantation & \\
Motor disorders & $35 / 32$ & $\mathrm{n} . \mathrm{s}$ \\
Urinary dysfunction & $50 / 27$ & $p=0.0126$ \\
Neuropathic pain & $13 / 2$ &
\end{tabular}

4.1. Limitations. Our study has some limitations due to the retrospective nature of data and the different evaluation scales used among the different articles. This did not allow further subgroup analyses (different MS types, different motor and urinary symptoms, and different pain locations).

\section{Conclusions}

The results of this systematic review suggest that SCS is effective in MS patients. Neuropathic pain and urinary dysfunction are significantly improved after SCS compared with motor disorders. Moreover, a proper stimulation trial is useful in increasing the number of patients responding to this treatment. Further studies with longer FU are needed to improve the patient selection, clarify the best timing to perform SCS in these patients, and better understand the potential loss of effectiveness of SCS over time.

\section{Data Availability}

The data used to support the findings of the study are available from the corresponding author upon request.

\section{Ethical Approval}

All procedures performed in studies involving human participants were in accordance with the ethical standards and with the 1964 Helsinki Declaration and its later amendments or comparable ethical standards. 


\section{Consent}

This is a systematic review of the literature, and therefore, consent is not required.

\section{Conflicts of Interest}

The authors declare that there are no conflicts of interest regarding the publication of this article.

\section{References}

[1] P. Flachenecker, T. Henze, and U. K. Zettl, "Spasticity in patients with multiple sclerosis - clinical characteristics, treatment and quality of life," Acta Neurologica Scandinavica, vol. 129, no. 3, pp. 154-162, 2014.

[2] C. Solaro, E. Trabucco, and M. Messmer Uccelli, "Pain and multiple sclerosis: pathophysiology and treatment," Current Neurology and Neuroscience Reports, vol. 13, no. 1, p. 320, 2013.

[3] J. M. Gelfand, "Multiple sclerosis and related disorders," Handbook of Clinical Neurology, vol. 122, pp. 269-290, 2014.

[4] V. Phé, E. Chartier-Kastler, and J. N. Panicker, "Management of neurogenic bladder in patients with multiple sclerosis," Nature Reviews Urology, vol. 13, no. 5, pp. 275-288, 2016.

[5] H. Abboud, E. Hill, J. Siddiqui, A. Serra, and B. Walter, "Neuromodulation in multiple sclerosis," Multiple Sclerosis Journal, vol. 23, no. 13, pp. 1663-1676, 2017.

[6] A. W. Cook and S. P. Weinstein, "Chronic dorsal column stimulation in multiple sclerosis. Preliminary report," New York State Journal of Medicine, vol. 73, no. 24, pp. 2868-2872, 1973.

[7] D. Moher, A. Liberati, J. Tetzlaff, D. G. Altman, and PRISMA Group, "Preferred reporting items for systematic reviews and meta-analyses: the PRISMA statement," PLoS Medicine, vol. 6, Article ID e1000097, 2009.

[8] J. A. Rosen and A. H. Barsoum, "Failure of chronic dorsal column stimulation in multiple sclerosis," Annals of Neurology, vol. 6, no. 1, pp. 66-67, 1979.

[9] R. F. Young and S. J. Goodman, "Dorsal spinal cord stimulation in the treatment of multiple sclerosis," Neurosurgery, vol. 5, no. 2, pp. 225-230, 1979.

[10] L. S. Illis, E. M. Sedgwick, and R. C. Tallis, "Spinal cord stimulation in multiple sclerosis: clinical results," Journal of Neurology, Neurosurgery \& Psychiatry, vol. 43, no. 1, pp. 1-14, 1980.

[11] J. Siegfried, Y. Lazorthes, and G. Broggi, "Electrical spinal cord stimulation for spastic movement disorders," Stereotactic and Functional Neurosurgery, vol. 44, no. 1-3, pp. 77-92, 1981.

[12] A. W. Cook, J. K. Taylor, and F. Nidzgorski, "Results of spinal cord stimulation in multiple sclerosis," Stereotactic and Functional Neurosurgery, vol. 44, no. 1-3, pp. 55-61, 1981.

[13] J. M. Waltz, W. H. Andreesen, and D. P. Hunt, "Spinal cord stimulation and motor disorders," Pacing and Clinical Electrophysiology, vol. 10, no. 1, pp. 180-204, 1987.

[14] K. Kumar, G. Hunter, and D. Demeria, "Spinal cord stimulation in treatment of chronic benign pain: challenges in treatment planning and present status, a 22 -year experience," Neurosurgery, vol. 58, no. 3, pp. 481-496, 2006.

[15] A. Compston and A. Coles, "Multiple sclerosis," The Lancet, vol. 372, no. 9648, pp. 1502-1517, 2008.

[16] N. Koch-Henriksen and P. S. Sørensen, "The changing demographic pattern of multiple sclerosis epidemiology," The Lancet Neurology, vol. 9, no. 5, pp. 520-532, 2010.
[17] J. Oh, A. Vidal-Jordana, and X. Montalban, "Multiple sclerosis: clinical aspects," Current Opinion in Neurology, vol. 31, no. 6, pp. 752-759, 2018.

[18] C. Solaro, G. Brichetto, M. P. Amato et al., "The prevalence of pain in multiple sclerosis: a multicenter cross-sectional study," Neurology, vol. 63, no. 5, pp. 919-921, 2004.

[19] N. Montano, S. Gaudino, C. Giordano et al., "Possible prognostic role of magnetic resonance imaging findings in patients with trigeminal neuralgia and multiple sclerosis who underwent percutaneous balloon compression: report of our series and literature review," World Neurosurgery, vol. 125, pp. e575-e581, 2019.

[20] N. Montano, A. Rapisarda, E. Ioannoni, and A. Olivi, "Microvascular decompression in patients with trigeminal neuralgia and multiple sclerosis: results and analysis of possible prognostic factors," Acta Neurologica Belgica, vol. 120, no. 2, pp. 329-334, 2020.

[21] D. A. Provenzano, J. R. Williams, G. Jarzabek, L. A. DeRiggi, and T. F. Scott, "Treatment of neuropathic pain and functional limitations associated with multiple sclerosis using an MRIcompatible spinal cord stimulator: a case report with two year follow-up and literature review," Neuromodulation: Technology at the Neural Interface, vol. 19, no. 4, pp. 406-413, 2016.

[22] G. Baranidharan, D. Edgar, B. Bretherton et al., "Efficacy and safety of $10 \mathrm{kHz}$ spinal cord stimulation for the treatment of chronic pain: a systematic review and narrative synthesis of real-world retrospective studies," Biomedicines, vol. 9, no. 2, p. 180, 2021. 